This item was submitted to Loughborough's Research Repository by the author.

Items in Figshare are protected by copyright, with all rights reserved, unless otherwise indicated.

\title{
Determination of residual stress and interface adhesion toughness of thin films by blisters
}

\section{PLEASE CITE THE PUBLISHED VERSION}

https://doi.org/10.1002/mdp2.60

\section{PUBLISHER}

(c) Wiley

\section{VERSION}

AM (Accepted Manuscript)

\section{PUBLISHER STATEMENT}

This is the peer reviewed version of the following article: YUAN, B. ... et al., 2019. Determination of residual stress and interface adhesion toughness of thin films by blisters. Material Design and Processing Communication, 1 (5), e60, which has been published in final form at https://doi.org/10.1002/mdp2.60. This article may be used for non-commercial purposes in accordance with Wiley Terms and Conditions for Use of Self-Archived Versions.

\section{LICENCE}

CC BY-NC-ND 4.0

\section{REPOSITORY RECORD}

Yuan, Bo, Christopher Harvey, Gary W. Critchlow, Rachel Thomson, and Simon Wang. 2019. "Determination of Residual Stress and Interface Adhesion Toughness of Thin Films by Blisters". figshare. https://hdl.handle.net/2134/37384. 


\title{
Title: Determination of residual stress and interface adhesion toughness of thin films by blisters
}

\section{Short running title: Properties of thin films by blisters}

\author{
Bo Yuan ${ }^{1}$, Christopher M. Harvey ${ }^{1}$, \\ Gary W. Critchlow ${ }^{2}$ and Rachel C. Thomson ${ }^{2}$, and Simon Wang ${ }^{1,3, *}$, \\ ${ }^{1}$ Department of Aeronautical and Automotive Engineering, Loughborough University, \\ Loughborough, Leicestershire LE11 3TU, UK \\ ${ }^{2}$ Department of Materials, Loughborough University, \\ Loughborough, Leicestershire LE11 3TU, UK \\ ${ }^{3}$ College of Mechanical and Equipment Engineering, \\ Hebei University of Engineering, Handan 056038, China
}

Keywords: thin film, residual stress, fracture toughness, spalling fracture, adhesion strength

*Corresponding author

Email addresses:

S.Wang@lboro.ac.uk (Simon Wang),

B.Yuan@lboro.ac.uk (Bo Yuan),

C.M.Harvey@lboro.ac.uk (Christopher M. Harvey),

R.C.Thomson@lboro.ac.uk (Rachel C. Thomson),

G.W.Critchlow@lboro.ac.uk (Gary W. Critchlow). 


\title{
Determination of residual stress and interface adhesion toughness of thin films by blisters
}

\begin{abstract}
Circular blisters and telephone cord blisters (TCBs) can spontaneously occur in thin films under constant bi-axial compressive residual stress. In this work, new mechanical models are used in conjunction with measurements of blister morphology parameters to determine the residual stress in films and the adhesion toughness at interfaces. These new models are based on the hypothesis that pockets of energy concentration (PECs) drive the nucleation and growth of blisters instead of buckling, as in the conventional models, since the thin films are under constant compressive residual stress. Predictions from the models are in excellent agreement with independent experimental data.
\end{abstract}

Keywords: thin film, residual stress, fracture toughness, spalling fracture, adhesion strength

\section{Introduction}

Thin-layer/thick-substrate composite material systems are ubiquitous in daily life. Thin layer materials exist in various forms such as films, coatings and oxidation scales. In this work, they are collectively called 'thin films'. Thin films perform various functions such as corrosion protection [1], thermal barriers [1], stretchable electrodes in electronics [2] and light trapping in solar cells [3]. Durability against delamination of composite material systems is a key design consideration, which is closely related to the residual stress in the film and the adhesion toughness at the interface. This work reports a method for both laboratory and in-situ measurements of the residual stress and adhesion toughness.

Film delamination from a substrate often occurs as blisters, which appear to develop spontaneously under constant compressive residual stresses. They are typically circular, straight or telephone cord blisters (TCBs). These blisters are conventionally and most-widely considered to be buckling-driven [4,5], that is, a pre-existing interface delamination without outward deflection is assumed with a size larger than the critical buckling dimension $[4,5]$. Experimental observations [6], however, show no such pre-existing delamination. Wang, Harvey et al. have recently proposed that spontaneous formation of blisters is driven by pockets of energy concentration (PECs) [7-9]. Predictions of blister morphology from these 
PEC-driven models [6-8] are in excellent agreement with independent experimental results. In this work, the measured morphology parameters are used to determine the compressive residual stress in thin films and the adhesion toughness at the interface. The mechanical models are briefly introduced in Section 2, and determination of residual stresses and adhesion toughness for specific cases are reported in Section 3. Conclusions are drawn in Section 4.

\section{Mechanical models}

\subsection{Circular blisters}

A circular blister with relevant geometry parameters is shown in Figure 1. The film material is assumed to be homogeneous and isotropic with the Young's modulus $E$ and Poisson's ratio $v$.

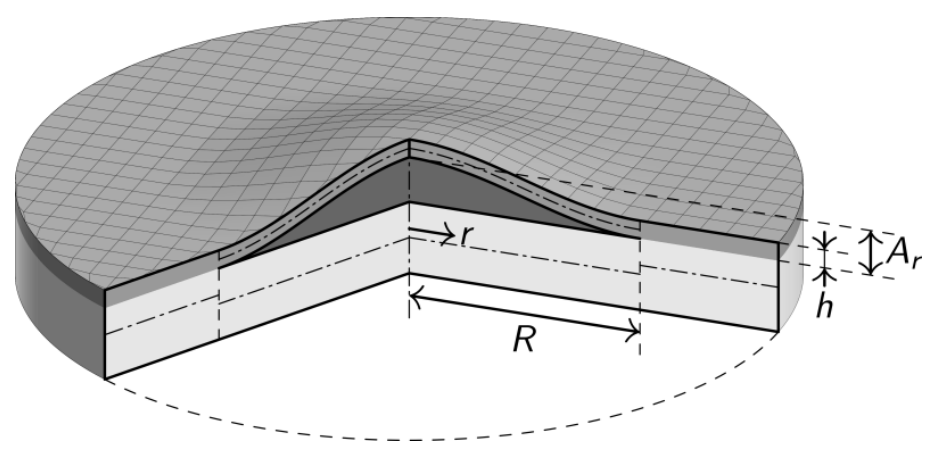

Figure 1. A circular blister.

According to the PECs theory in Ref. [7,8], the spontaneous formation of a blister is determined by the parameter $\Omega$, which represents the ratio between the plane-strain residual strain energy density in the thin film and the adhesion toughness $G_{\mathrm{c}}$ at the interface, that is,

$$
\Omega=\frac{h \sigma_{0}^{2}\left(1-v^{2}\right)}{2 E G_{\mathrm{c}}},
$$

where $\sigma_{0}$ is the bi-axial compressive residual stress. The mechanical condition for the of a circular blister is $\Omega>3 / 2$ [7,8]. It is worth noting that the value of $\Omega$ depends on the film thickness $h$, the residual stress $\sigma_{0}$ and the adhesion toughness $G_{\mathrm{c}}$ for a given material. Using measurements of film thickness $h$ and blister height $A_{r}$ at spallation, as shown in Figure 1, the parameter $\Omega$ can be calculated as 


$$
\Omega=3\left(\frac{A_{r}}{8 h}+\frac{h}{A_{r}}\right)^{2} .
$$

Then the residual compressive stress $\sigma_{0}$ is obtained by using $\Omega$ and the measured blister spallation radius $R$, as follows:

$$
\sigma_{0}=\frac{\pi^{2} E \Omega}{3\left(1-v^{2}\right)}\left(\frac{h}{R}\right)^{2}\left[1-\left(1-\frac{3}{2 \Omega}\right)^{1 / 2}\right] .
$$

If $\Omega>>3 / 2$ then the residual stress $\sigma_{0}$ can be determined with $R$ only, that is,

$$
\sigma_{0}=\frac{\pi^{2} E}{4\left(1-v^{2}\right)}\left(\frac{h}{R}\right)^{2}
$$

The adhesion toughness $G_{\mathrm{c}}$ at interface is then determined using Eq. (1).

\subsection{Telephone cord blisters (TCB)}

Figure 2 shows a TCB consisting of the fully-developed part and the nose part. The fullydeveloped part has width $2 R$, height $A_{x}$, transverse amplitude $A_{y}$ and wavelength $\lambda$. These are the four morphology parameters of a TCB.

(a)

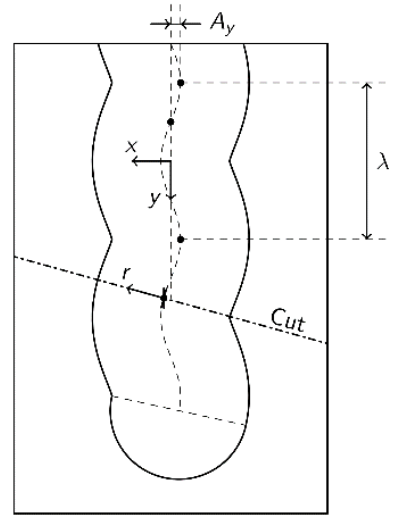

(b)

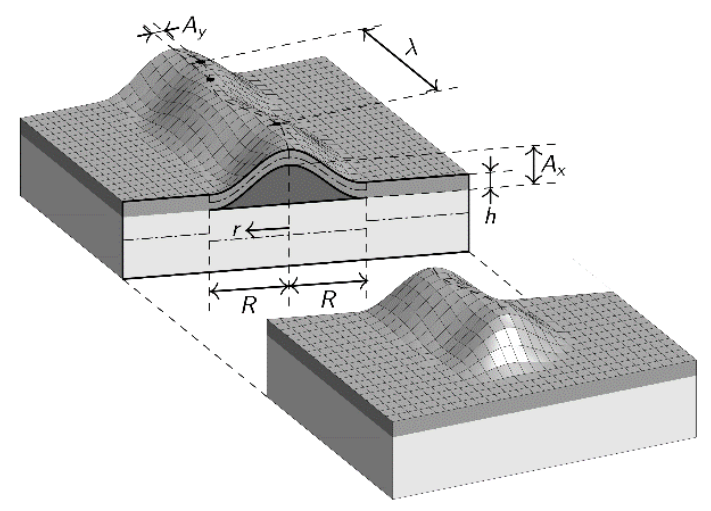

Figure 2. A telephone cord blister: (a) Top view and (b) 3D view of the cut (shown in a) along the $r$-axis perpendicular to the sinusoidal centerline of the blister.

Yuan, Harvey et al. [9] have developed a completely-analytical model to determine the four morphology parameters based on the PECs theory $[7,8]$. The theoretical predictions are again in excellent agreement with extensive independent experimental data. Now, in this work, the calculation is reversed to determine the residual stresses in the film and the adhesion toughness at the interface by using measured morphology parameters. By using 
measurements of the TCB height $A_{x}$ the parameter $\Omega$ is still given by Eq. (2), but with the subscript $x$-coordinate swapped for the $r$-coordinate. Then the residual stress $\sigma_{0}$ is obtained as

$$
\sigma_{0}=\frac{\pi^{2} E \Omega}{3\left(1-v^{2}\right)}\left(\frac{h}{R}\right)^{2}\left[1+\left(1-\frac{3}{2 \Omega}\right)^{1 / 2}\right] .
$$

The adhesion toughness at the interface $G_{\mathrm{c}}$ is still determined using Eq. (1).

It is worth noting that parameter $\Omega$ can also be determined by using the measured wavelength-to-width ratio, that is,

$$
\Omega=\frac{3}{2}\left\{1-\left(\frac{v-1}{v+[\lambda /(2 R)]^{2}}\right)^{2}\right\}^{-1} .
$$

\section{Determination of residual stresses and adhesion toughness}

The first case concerns the determination of residual stresses in alumina scales grown by oxidation of FeCrAl alloy at high temperature [6]. Circular blisters spontaneously formed under constant bi-axial compressive residual stresses and at room temperature after cooling from high oxidation temperature. Their sizes were much smaller than the critical buckling dimensions during both the nucleation and early-growth stages. After a period of unstable growth, many blisters spalled off. In this work, the spallation radius measurements are used to determine the residual stresses. Four, two and one blisters were measured in alumina scales of thickness $4.9 \mu \mathrm{m}, 6.2 \mu \mathrm{m}$ and $8.0 \mu \mathrm{m}$, respectively. The average spallation radii for each alumina scale thickness are $75 \mu \mathrm{m}, 97.5 \mu \mathrm{m}$ and $122.5 \mu \mathrm{m}$, respectively. The respective blister heights are not available. The Young's modulus and Poisson's ratio of the alumina scales is $E=400 \mathrm{GPa}$ and $v=0.25$, respectively. Since the blister heights are not available the parameter $\Omega$ can not be determined using Eq. (2). The residual compressive stresses $\sigma_{0}$ are therefore predicted using Eq. (4), and comparisons with the experimental results are presented in Table 1. It is seen that an excellent agreement has been achieved between the prediction and experimental values. This indicates that $\Omega$ is much larger than $3 / 2$. 
Table 1. Combined comparison of the residual compressive stresses $\sigma_{0}$ in alumina scales.

\begin{tabular}{ccc}
\hline Film thickness $[\mu \mathrm{m}]$ & $\sigma_{0}[\mathrm{GPa}]$, Eq. (4) & $\sigma_{0}[\mathrm{GPa}]$ (measured in Ref. [6]) \\
\hline 4.9 & 4.49 & 4.46 \\
6.2 & 4.26 & 4.45 \\
8.0 & 4.49 & 4.31 \\
\hline
\end{tabular}

The second case concerns the determination of residual stresses in tungsten film and its adhesion toughness at the interface with silica substrate. The TCB morphology parameters in Ref. [10] are used. The Young's modulus and Poisson's ratio of the tungsten films are taken to be $E=411 \mathrm{GPa}$ and $v=0.28$, respectively. There are four groups of specimens with different film thicknesses. The measurements of the TCB width $2 R$ and height $A_{x}$ were performed at the curved section and straight section respectively, as shown in Figure 3.

(a)

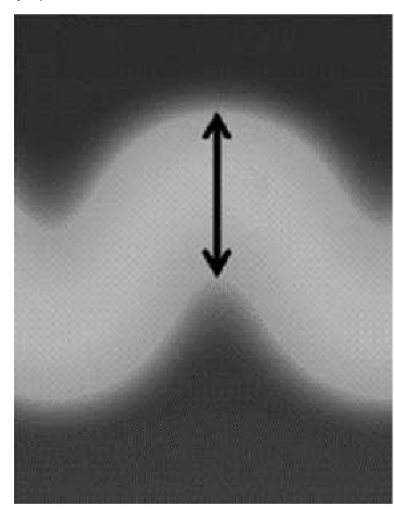

(b)

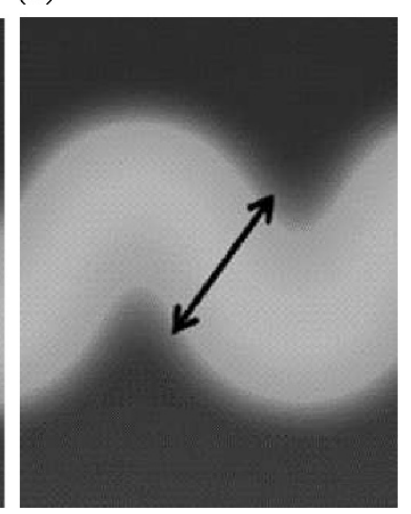

Figure 3. Telephone cord blister morphology measurement at (a) curved section and (b) straight section [10].

The values of $\Omega$ for each TCB are calculated using Eq. (2) with the measured value of $A_{x}$ , and then the compressive residual stresses $\sigma_{0}$ and interface adhesion toughnesses $G_{\mathrm{c}}$ are obtained using Eqs. (5) and (1), respectively. The results are recorded in Table 2, in which each group of results is for a different film thickness. It is worth noting that TCBs can form at different values of $\Omega>1.5$ which depends on the film thickness $h$, the residual stress $\sigma_{0}$ and the adhesion toughness $G_{\mathrm{c}}$ for a given material [7,8]. It is observed that all $\Omega$ values in Table 2 are larger than 1.5, which satisfies the formation condition of TCBs, that is, $\Omega>1.5[7,8]$. Note that the second group of specimens strictly obeys this rule. The determined compressive residual stresses $\sigma_{0}$ and interface adhesion toughness $G_{\mathrm{c}}$ at the curved and straight sections in each group are very close to each other as expected. This demonstrates the validity of the 
present method. The variations of residual stresses $\sigma_{0}$ and interface adhesion toughness $G_{\mathrm{c}}$ in different groups show the effect of film thickness $h$. The critical mode I and mode II adhesion toughnesses, $G_{\text {Ic }}$ and $G_{\text {IIc }}$, can then be determined by using a mixed mode partition theory $[7,8]$.

Table 2. Theoretical predictions of the residual compressive stresses $\sigma_{0}$ and interface adhesion toughness $G_{\mathrm{c}}$ with the morphologies of telephone cord blisters from Ref. [10].

\begin{tabular}{|c|c|c|c|c|c|c|}
\hline \multicolumn{4}{|c|}{ Experimental measurements [10] } & \multicolumn{3}{|c|}{ Theoretical predictions } \\
\hline Section & $h[\mathrm{~nm}]$ & $R[\mu \mathrm{m}]$ & $A_{x}[\mu \mathrm{m}]$ & $\Omega$, Eq. (2) & $\begin{array}{c}\sigma_{0}[\mathrm{GPa}], \\
\text { Eq. }(5)\end{array}$ & $\begin{array}{c}G_{\mathrm{c}}\left[\mathrm{Nm}^{-2}\right], \\
\text { Eq. (1) }\end{array}$ \\
\hline Curved & & 8.11 & 0.847 & 4.15 & 1.67 & 0.08 \\
\hline Straight & 100 & 7.4 & 0.787 & 3.70 & 1.76 & 0.09 \\
\hline Averaged & & 7.76 & 0.817 & 3.93 & 1.72 & 0.09 \\
\hline Curved & & 5.4 & 0.67 & 1.54 & 3.63 & 1.91 \\
\hline Straight & 200 & 5.16 & 0.633 & 1.52 & 3.72 & 2.05 \\
\hline Averaged & & 5.28 & 0.652 & 1.53 & 3.68 & 1.98 \\
\hline Curved & & 17.5 & 1.43 & 2.72 & 1.10 & 0.11 \\
\hline Straight & 225 & 17.2 & 1.36 & 2.54 & 1.05 & 0.11 \\
\hline Averaged & & 17.35 & 1.40 & 2.63 & 1.08 & 0.11 \\
\hline Curved & & 20 & 1.73 & 2.40 & 1.28 & 0.23 \\
\hline Straight & 300 & 18.8 & 1.47 & 2.00 & 1.12 & 0.21 \\
\hline Averaged & & 19.4 & 1.60 & 2.20 & 1.20 & 0.22 \\
\hline
\end{tabular}

\section{Conclusion}

Spontaneous formation of circular and telephone cord blisters in thin films can occur when the parameter $\Omega$, that is, the ratio between the plane-strain residual strain energy density and the adhesion toughness, is larger than $3 / 2$. By measuring the blister morphology parameters, the authors' PEC-driven mechanical models [7-9] provide an accurate method to determine the residual stresses in a thin film and the adhesion toughness at the interface between the film and its substrate. This method is of considerable value as it can be used in both laboratory tests and with in-situ measurements.

\section{References}

[1] N.P. Padture, Thermal Barrier Coatings for Gas-Turbine Engine Applications, Science. 296 (2002) 280-284. doi:10.1126/science.1068609.

[2] J.A. Rogers, T. Someya, Y. Huang, Materials and Mechanics for Stretchable Electronics, Science. 327 (2010) 1603-1607. doi:10.1126/science.1182383.

[3] M.L. Brongersma, Y. Cui, S. Fan, Light management for photovoltaics using high- 
index nanostructures, Nat. Mater. 13 (2014) 451-460. doi:10.1038/nmat3921.

[4] J.W. Hutchinson, Z. Suo, Mixed Mode Cracking in Layered Materials, in: Adv. Appl. Mech., 1991: pp. 63-191.

[5] M.W. Moon, H.M. Jensen, J.W. Hutchinson, K.H. Oh, A.G. Evans, The characterization of telephone cord buckling of compressed thin films on substrates, J. Mech. Phys. Solids. 50 (2002) 2355-2377. doi:10.1016/S0022-5096(02)00034-0.

[6] V.K. Tolpygo, D.R. Clarke, Spalling failure of $\alpha$-alumina films grown by oxidation. II. Decohesion nucleation and growth, Mater. Sci. Eng. A. 278 (2000) 151-161. doi:10.1016/S0921-5093(99)00582-1.

[7] S. Wang, C.M. Harvey, B. Wang, Room temperature spallation of $\alpha$-alumina films grown by oxidation, Eng. Fract. Mech. $178 \quad$ (2017) 401-415. doi:10.1016/j.engfracmech.2017.03.002.

[8] C.M. Harvey, B. Wang, S. Wang, Spallation of thin films driven by pockets of energy concentration, Theor. Appl. Fract. Mech. 92 (2017) 1-12. doi:10.1016/j.tafmec.2017.04.011.

[9] B. Yuan, C.M. Harvey, R.C. Thomson, G.W. Critchlow, D.S. Rickerby, S. Wang, Spontaneous formation and morphology of telephone cord blisters in thin films: The $\Omega$ formulae, In review.

[10] M.J. Cordill, D.F. Bahr, N.R. Moody, W.W. Gerberich, Adhesion measurements using telephone cord buckles, Mater. Sci. Eng. A. 443 (2007) 150-155. doi:10.1016/j.msea.2006.08.027. 